\title{
MGS TES Results: Atmospheric Structure, Aerosols, and Dynamics
}

\author{
Barney J. Conrath \\ Cornell University, Ithaca $N Y$, USA \\ John C. Pearl, Michael D. Smith \\ NASA/GSFC, Greenbelt MD, USA \\ Philip R. Christensen \\ Arizona State University, Tempe AZ, USA
}

\begin{abstract}
Thermal emission spectra from the MGS TES instrument permit retrieval of atmospheric properties that are of interest in the study of Martian meteorology, including temperature, dust, and water ice. Examples of analyses of zonal mean meridional thermal sructure for equinox and solstice conditions are presented.
\end{abstract}

\section{Introduction}

The Thermal Emission Spectrometer (TES) carried on the Mars Global Surveyor (MGS) spacecraft obtains measurements of the Martian thermal emission spectrum between 200 and $1600 \mathrm{~cm}^{-1}$. The instrument is a Michelson interferometer with selectable nominal spectral resolutions of 5 and $10 \mathrm{~cm}^{-1}$. Interferograms are Fourier transformed on board the spacecraft prior to transmission to the Earth. The focal plane contains a $2 \times 3$ detector array with an angular resolution of 8.3 milliradians per pixel. A pointing mirror permits viewing of the nadir as well as forward and aft limbs on command. Calibration of the interferometer is accomplished by periodically viewing cold space and an onboard blackbody of known temperature. The large majority of spectra thus far have been taken in the $10 \mathrm{~cm}^{-1}$ resolution mode, yielding a set of six spectra every 2 seconds. At this resolution, a noise equivalent radiance of about $2 \times 10^{-8} \mathrm{~W}$ $\mathrm{cm}^{-2}$ ster $^{-1} / \mathrm{cm}^{-1}$ is achieved. A detailed description of the instrument and its use is given by Christensen et al. (1992).

In this report, only results obtained since the beginning of the MGS mapping orbit phase will be discussed. The near-polar orbit is solar-synchronous at 2:00 a.m. and 2:00 p.m. local Mars time. The approximately 2-hour orbital period permits about 12 orbits of data to be obtained per martian day, resulting in an orbit-to-orbit spacing in ground track longitude at the equator of about 29 degrees. Since there is an approximately 12-degree advance in longitude per day in the groundtrack pattern, the resolution in longitude can be improved at the expense of time-smearing by combining several days of data. 
Although the TES spectrometer was designed primarily for surface studies, it has also proven to be an excellent instrument for the study of the Martian atmosphere (Christensen et al. 1998; Conrath et al. 2000; Smith et al. 2000). A selection of atmospheric results are presented here with emphasis on the retrieval of thermal structure and its use in diagnostic studies of the Martian atmosphere. In the following section, the characteristics of the Martian spectra obtained by TES are discussed along with approaches used in extracting information. Retrieved thermal structure and its implications for dynamics are presented in Section 3, and the present state of the analysis is summarized.

\section{TES Spectra}

The majority of the spectra taken by TES are in the nadir viewing mode. In this mode, each of the six detectors has a $3 \mathrm{~km} \times 3 \mathrm{~km}$ beam footprint on the Martian surface with the wide dimension across the ground track. The three $\mathrm{km}$ ground track speed results in a $6 \mathrm{~km}$ along track smearing with the $10 \mathrm{~cm}^{-1}$ spectral resolution mode. In addition to the nadir spectra, multifootprint sequences of limb-viewing spectra are taken about every 10 degrees of latitude with measurements extending from the planetary surface to altitudes greater than $100 \mathrm{~km}$. In the nadir spectra, the atmospheric features appear in absorption because the relatively cool atmosphere is viewed against the warmer planetary surface.

A parameter of primary importance in the study of the meteorology of the Martian atmosphere is the three dimensional thermal structure and its evolution with time and season. In addition, atmospheric temperature profiles are required for the quantitative retrieval of other atmospheric parameters from thermal emission spectra. Measurements within the $667 \mathrm{~cm}^{-1} \mathrm{CO}_{2}$ band are inverted to obtain temperature as a function of barometric pressure under the assumption that $\mathrm{CO}_{2}$ is uniformly mixed throughout the Martian atmosphere. Spectral information across the band is used to obtain profiles in the lower part of the atmosphere from nadir measurements, while a combination of spectral and geometric information from the limb spectra provides temperatures at higher atmospheric levels. At intervals of about 10 degrees of latitude along the ground track of the spacecraft, limb and nadir results can be combined to obtain temperature as a function of pressure from the surface up to about $0.008 \mathrm{mbar}$ (nominally, about $65 \mathrm{~km}$ above the 6.1 mbar surface) .

Once the temperature profile has been obtained, it is then possible to use the spectral features of dust and ice to infer the total infrared optical depths of those constituents. Limb spectra can also be used to obtain vertical information on these parameters.

\section{Results}

From the beginning of the mapping phase of the mission to the present writing, in excess of 170,000 composite limb-plus-nadir temperature profiles have been obtained. This data set is being used extensively to study Martian meteorology. Here we present examples from one analysis that has been carried out. 

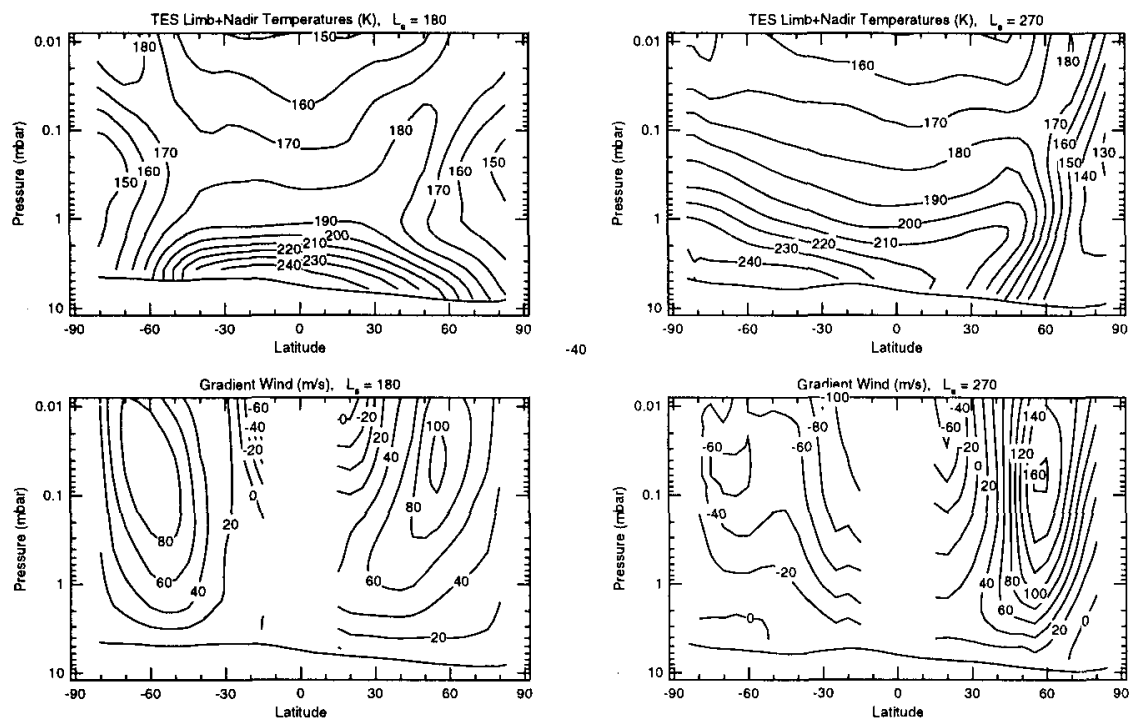

Figure 1. Zonal mean meridional temperature (upper panels) and thermal wind (lower panels) cross sections. The left hand set represents equinox conditions while the right hand set illustrates solstice conditions.

A zero order characterization of the thermal structure of a planetary atmosphere consists of the zonal (longitude) mean meridional temperature cross section and its evolution with season. Such temperature cross sections constructed from the TES results are shown in the upper panels of Figure 1. Temperature profiles from five-day periods were averaged to ensure adequate sampling in longitude, while daytime and nighttime data were combined to remove possible effects of the migrating diurnal atmospheric tidal modes. The north-south temperature gradients on constant pressure surfaces can be used to calculate the zonal (east-west) component of the wind field. These so-called thermal winds are obtained under the assumption of hydrostatic and gradient wind balance, and are generally valid on a rapidly rotating planet except near the equator. Since only the vertical wind shear is obtained in this way, it was assumed that the wind speed vanishes at the planetary surface in constructing the wind fields in the lower panels of Figure 1. The left-hand panels in Figure 1 are from the time of the northern hemisphere autumnal equinox $\left(\mathrm{L}_{s}=180^{\circ}\right)$. If no dynamic processes were active in the atmosphere, the temperature field should be in radiative equilibrium; departures from radiative equilibrium can be diagnostic of atmospheric motion. Inspection of the equinox cross section indicates that it cannot be in radiative equilibrium because of the temperature maxima aloft in both hemispheres. This departure from equilibrium results primarily from the meridional (Hadley) circulation with rising motion due to solar heating at low latitudes and descending motion at mid and high latitudes in both hemispheres, with the latter producing compressional heating. The zonal wind (lower left panel) shows broad eastward jets in both hemispheres associated with the 
strong high latitude temperature gradients. Westward winds are observed aloft at lower latitudes.

Conditions at the time of the southern hemisphere summer solstice $\left(\mathrm{L}_{s}=\right.$ $270^{\circ}$ ) are shown in the right hand-panels of Figure 1. Note that the atmospheric temperatures increase monotonically from the equator to the south pole in response to the maximum in integrated solar heating at high latitudes at this season. This structure is qualitatively quite different from the terrestrial atmosphere where the presence of the ocean heat resovoir does not permit large departures of the temperature from the annual mean. At this time there is primarily a single Hadley cell with rising motion at mid to high southern latitudes and decending motion producing compressional heating in the north. This heating in the north, combined with radiative cooling in the northern winter polar region, results in an intense temperature gradient with latitude that provides the thermal wind balance for the intense eastward circumpolar jet seen in the winter hemisphere in the lower right hand panel. The strong deep cross-equatorial Hadley circulation inferred on Mars is not observed in the terrestrial atmosphere. The winter polar front and associated circumpolar jet are found on both planets, but that on Mars is substantially deeper and more intense.

Because it is optically active in both the visible and thermal infrared, dust is an important factor in the energetics of the atmosphere and can profoundly affect the circulation, especially during dust storm conditions. TES spectra have been used to map the dust optical depth and to establish its infrared optical properties. Several regional dust storms and their evolution have been observed, and their effects on the thermal structure have been documented. Water ice clouds have also been mapped and the seasonal evolution of their distribution studied. The details of these investigations cannot be included here, but the interested reader is referred to Smith et al. (2000), Conrath et al. (2000), and Pearl et al. (2000).

\section{References}

Christensen, P. R., et al. 1992, JGR, 97, 7719

Christensen, P.R., et al. 1998, Science, 279, 1692

Conrath, B. J., Pearl, J. C., Smith, M. D., Maguire, W. C., Dason, S., Kaelberer, M. S., \& Christensen, P. R. 2000, JGR, 105, 9509

Pearl, J.C., Conrath, B. J., Smith, M.S., Bandfield, J.L. \& Christensen, P.R. $2000, \mathrm{JGR}$, in press

Smith, M.D., Pearl, J. C., Conrath, B. J., \& Christensen, P. R. 2000, JGR, 105, 9539 